Acta Crystallographica Section D

\section{Biological Crystallography}

ISSN 0907-4449

Isabel Bento, ${ }^{a}$ Cristina Peixoto, ${ }^{b}$ Vjacheslav N. Zaitsev ${ }^{c}$ and Peter F. Lindley ${ }^{a, d}$.

a Instituto de Tecnologia Química e Biológica, Universidade Nova de Lisboa, Apartado 127, 2781-901 Oeiras, Portugal, 'baboratory of Animal Cell Technology, Instituto de Biologia Experimental e Tecnológica, Apartado 12, 2781-901 Oeiras, Portugal, 'cCentre for Biomolecular Sciences, University of St Andrews, North Haugh, St Andrews KY16 9ST, Scotland, and ${ }^{\mathbf{d}}$ Department of Crystallography, Birkbeck College, University of London, Malet Street, London WC1E 7HX, England

Correspondence e-mail:

lindley.p@googlemail.com

\title{
Ceruloplasmin revisited: structural and functional roles of various metal cation-binding sites
}

The three-dimensional molecular structure of human serum ceruloplasmin has been reinvestigated using X-ray synchrotron data collected at $100 \mathrm{~K}$ from a crystal frozen to liquidnitrogen temperature. The resulting model, with an increase in resolution from 3.1 to $2.8 \AA$, gives an overall improvement of the molecular structure, in particular the side chains. In addition, it enables the clear definition of previously unidentified $\mathrm{Ca}^{2+}$-binding and $\mathrm{Na}^{+}$-binding sites. The $\mathrm{Ca}^{2+}$ cation is located in domain 1 in a configuration very similar to that found in the activated bovine factor $\mathrm{Va}$. The $\mathrm{Na}^{+}$sites appear to play a structural role in providing rigidity to the three protuberances on the top surface of the molecule. These features probably help to steer substrates towards the mononuclear copper sites prior to their oxidation and to restrict the size of the approaching substrate. The trinuclear copper centre appears to differ from the room-temperature structure in that a dioxygen moiety is bound in a similar way to that found in the endospore coat protein $\operatorname{Cot} \mathrm{A}$ from Bacillus subtilis.

\section{Introduction}

Human ceruloplasmin, hCP, is a member of the multi-copper oxidase family of enzymes that includes ascorbate oxidase and the laccases. It was first isolated in 1944 (Holmberg, 1944) and has a molecular weight of some $132 \mathrm{kDa}$, being comprised of a single polypeptide chain of 1046 amino-acid residues with a carbohydrate content of between $7 \%$ and $8 \%$ (Takahashi et al., 1984). The first X-ray structural study of human ceruloplasmin was reported in 1996 (Zaitseva et al., 1996) and confirmed that the molecule is comprised of six cupredoxintype domains arranged in a triangular array. It also showed that there are six integral copper ions, three of which form a trinuclear cluster at the interface of domains 1 and 6, whilst the remainder are arranged in three mononuclear sites, one each in domains 2, 4 and 6. Each of the mononuclear copper ions is coordinated to a cysteine and two histidine residues and those in domains 4 and 6 also coordinate weakly to a methionine residue; in domain 2, the methionine is replaced by a leucine residue at a van der Waals contact distance from the cation. The arrangements of the trinuclear centre and the mononuclear copper ion in domain 6 are essentially the same as that found in ascorbate oxidase (Messerschmidt et al., 1992) and other members of the laccase family (Lindley, 2001; Nakamura \& Go, 2005), strongly suggesting an oxidase role for ceruloplasmin in the plasma. The multi-functional nature
Received 11 October 2006

Accepted 18 November 2006

PDB Reference: ceruloplasmin, 2j5w, r2j5wsf.
C 2007 International Union of Crystallography Printed in Denmark - all rights reserved 
Table 1

Data-collection statistics for human ceruloplasmin.

Values in parentheses are for the highest resolution shell (2.95-2.80 А).

\begin{tabular}{ll}
\hline Beamline at ESRF & ID14-4 \\
Wavelength $(\AA)$ & 0.9393 \\
Crystal-to-detector distance $(\mathrm{mm})$ & 250 \\
Resolution $(\AA)$ & $75.3-2.8$ \\
Temperature $(\mathrm{K})$ & 100 \\
Space group & $P 3_{2} 21$ \\
Unit-cell parameters $(\AA)$ & $a=209.14, c=82.75$ \\
Mosaicity $\left({ }^{\circ}\right)$ & 0.3 \\
Oscillation range $\left(^{\circ}\right)$ & 60.00 \\
Oscillation angle $\left({ }^{\circ}\right)$ & 0.25 \\
No. of unique $h k l$ & $51383(7464)$ \\
Completeness $(\%)$ & $100.0(100.0)$ \\
$I / \sigma(I)$ & $6.4(2.3)$ \\
$\left.R_{\text {merge }}\right)$ & $0.074(0.319)$ \\
Multiplicity & $3.7(3.8)$ \\
\end{tabular}

of ceruloplasmin has been the subject of several reviews (see for example, Laurie \& Mohammed, 1980; Ryden, 1984; Lindley et al., 1999; Bielli \& Calabrese, 2002), but despite this structural information it was still not possible to define the precise function(s) of hCP.

Further structural studies involving the binding of the cations $\mathrm{Co}^{2+}, \mathrm{Fe}^{2+}$ and $\mathrm{Fe}^{3+}$ (Lindley et al., 1997) added substantial weight to the argument that ceruloplasmin has an oxidase role and indicated a putative mechanism for oxidase activity. They also provided a structural explanation regarding how mutations in the ceruloplasmin gene leading to the expression of a truncated form of the protein could lead to systemic haemosiderosis (Yoshida et al., 1995). A ferroxidase role of the enzyme in plasma therefore appears to have been substantiated and in this context ceruloplasmin may serve to assist the release of iron from cells prior to uptake of the metal by the transferrin (Frieden \& Hsieh, 1976; Lindley et al., 1999). Another series of structural studies (Zaitsev et al., 1999) located the binding sites for an inhibitor, azide, and various amine substrates. Surprisingly, it was found that there are two distinct sites for amine substrates. Aromatic diamines appear to bind near the bottom of domain 4 , close to an arrangement of tryptophan, methionine and histidine residues that is unique to this domain and remote from the mononuclear copper centre within it. Biogenic amines, such as norepinephrine, epinephrine, serotonin and dopa, bind at a site in domain 6 that is very close to the site used for cation oxidation. On the other hand, (+)-lysergic acid diethylamine (LSD) appears to bind close to the glycan moiety at Asn119 in domain 1.

However, all these structural studies have been severely hampered by the limited resolution of the X-ray data, which is of $3.0 \AA$ or worse. The protein is large in size, with some 8500 non-H atoms, and therefore tends to show weak diffraction of $\mathrm{X}$-rays. In addition, the trigonal crystals used for the X-ray data collection have a high solvent content, approaching $70 \%$, and are very sensitive at ambient temperatures to radiation damage, particularly when using the high-brilliance synchrotron X-ray sources that are necessary to obtain even mediumresolution data. Many attempts have been made to cryo-freeze
hCP crystals to temperatures approaching that of liquid nitrogen, but the high solvent content and sensitivity of the protein to chemical denaturation present real problems. However, recently a set of conditions has been found, as described in $\$ 2$, that has enabled the growth of crystals that can be cryo-frozen. The size of these crystals still needs to be improved, but is has been possible to collect X-ray data to $2.8 \AA$ using the European Synchrotron Radiation Facility at Grenoble, France. This modest increase in resolution, together with the cryotemperature, which minimizes the thermal motion of the atoms in the crystal, has enabled further X-ray studies resulting in new structural information on the $\mathrm{hCP}$ molecule, including the identification of $\mathrm{Ca}^{2+}$-binding and $\mathrm{Na}^{+}$-binding sites; these findings are reported here.

\section{Experimental}

\subsection{Chromatographic purification, crystallization and data collection}

Samples of hCP were obtained as described previously (Moshkov et al., 1979). However, prior to crystallization a final purification stage was undertaken using size-exclusion chromatography. The protein, at a concentration of approximately $100 \mathrm{mg} \mathrm{ml}^{-1}$, was loaded onto a HiPrep 16/30 Sephacryl S-300 column (GE Healthcare, USA) with a flow rate of $0.7 \mathrm{ml} \mathrm{min}^{-1}$ after the column had been equilibrated with five column volumes of $0.1 \mathrm{M}$ sodium acetate buffer with $100 \mathrm{mM}$ $\mathrm{NaCl}$. Elution was monitored online at 280 and $610 \mathrm{~nm}$ and the peaks were recovered and analyzed by SDS-PAGE. All chromatographic procedures were performed using an ÄKTA explorer 10S system (GE Healthcare) at $277 \mathrm{~K}$. The samples recovered after this 'polishing' stage were loaded onto NuPage (Invitrogen, UK) precast gradient 4-12\% acrylamide gels and analyzed using the Simply Blue Safe Stain (Invitrogen, UK). The total protein content was determined by the bicinchoninic acid (BCA) assay using a commercial kit (Pierce, USA) with bovine serum albumin as a standard.

$\mathrm{hCP}$ was crystallized by the hanging-drop vapour-diffusion technique using a $4 \mu \mathrm{l}$ total drop volume with $50 \%$ protein at a concentration of $50 \mathrm{mg} \mathrm{ml}^{-1}$ and a precipitant solution containing 8\% PEG 20K, 8\% PEG MME 550, $0.1 M$ sodium acetate, $0.2 M$ sodium formate $\mathrm{pH}$ 5.6. Small crystals of $0.15 \times$ $0.02 \times 0.08 \mathrm{~mm}$ in size were obtained at $277 \mathrm{~K}$ within a few days and the addition of $23 \%$ glycerol enabled these to be frozen to liquid-nitrogen temperature.

Diffraction data were collected to $2.8 \AA$ resolution under cryogenic conditions (100 K) at ESRF beamline ID14-4 with a wavelength of $0.9393 \AA$. All data were processed with MOSFLM (Leslie, 1992) and merged with the SCALA package from the $C C P 4$ program suite (Collaborative Computational Project, Number 4, 1994). Crystallographic data and data-collection statistics are summarized in Table 1.

\subsection{Structure determination and refinement}

The crystal structure was elucidated by the molecularreplacement technique using the program MOLREP (Vagin \& 
Table 2

Refinement and quality of refined model.

\begin{tabular}{|c|c|}
\hline \multicolumn{2}{|l|}{ Refinement } \\
\hline No. of protein atoms & 8338 \\
\hline No. of solvent atoms & 341 \\
\hline No. of heteroatoms & 14 \\
\hline No. of glycan units & $2 \mathrm{NAG}$ \\
\hline No. of glycerols & 2 \\
\hline \multicolumn{2}{|l|}{ Final $R$ factors } \\
\hline Working + test set & 0.178 \\
\hline Working & 0.175 \\
\hline Final free $R$ factor (5.1\% of reflections) & 0.228 \\
\hline \multicolumn{2}{|l|}{ Mean $B$ values $\left(\AA^{2}\right)$} \\
\hline Protein & 48.9 \\
\hline Solvent & 44.1 \\
\hline Overall & 48.8 \\
\hline \multicolumn{2}{|l|}{ ESU $(\AA)$ based on } \\
\hline Free $R$ value & 0.27 \\
\hline Maximum likelihood & 0.19 \\
\hline \multicolumn{2}{|l|}{ Correlation coefficients } \\
\hline$F_{\mathrm{o}}-F_{\mathrm{c}}$ & 0.95 \\
\hline Free & 0.91 \\
\hline \multicolumn{2}{|l|}{ Distance deviations $\dagger$} \\
\hline Bond distances $(\AA)$ & $0.011(0.022)$ \\
\hline Bond angles $(\AA)$ & $1.505(1.940)$ \\
\hline Planar groups $(\AA)$ & $0.004(0.020)$ \\
\hline Chiral volume deviation $\left(\AA^{3}\right)$ & $0.224(0.200)$ \\
\hline \multicolumn{2}{|l|}{ Quality of model $¥$} \\
\hline Overall $G$ factor & -0.04 \\
\hline \multicolumn{2}{|c|}{ Ramachandran analysis, number and $\%$ of residues in } \\
\hline Favourable regions & $749(86.7 \%)$ \\
\hline Additional regions & $109(12.6 \%)$ \\
\hline Generous regions & $5(0.6 \%)$ \\
\hline Disallowed regions & $1(0.1 \%)$ \\
\hline
\end{tabular}

$\dagger$ Values in parentheses are r.m.s. deviations from target values. $\ddagger$ The $G$ factor and Ramachandran analysis were determined using PROCHECK (Laskowski et al., 1993). Regions 18-32, 340-350 and 703-709 were omitted.

Teplyakov, 1997) from the CCP4 program suite. The roomtemperature structure of hCP (PDB code 1kcw; Zaitseva et al., 1996) was used as the search model with all the copper ions and sugar residues removed. Only one solution was evident, with a signal-to-noise ratio of 16.8 compared with 7.3 for the next highest solution and with an $R$ factor of 0.40 and a correlation coefficient of 0.58 . Refinement was performed using REFMAC (Murshudov et al., 1999) interspersed with manual correction of the model and stereochemical parameters using a combination of the $O$ (Jones et al., 1991) and Coot (Emsley \& Cowtan, 2004) computer-graphics packages and difference electron-density maps with $\left(F_{\mathrm{o}}-F_{\mathrm{c}}\right)$ and $\left(2 F_{\mathrm{o}}-F_{\mathrm{c}}\right)$ coefficients weighted according to the scheme devised by Read (1986). Metal cations were identified on the basis of chemical probability and the behaviour of their thermal isotropic parameters during refinement. For the various types of metal ions, restraints were used according to the standard CCP4 libraries. In the case of the dioxygen molecule between the two type 3 copper ions, OMIT maps (omitting one or both $\mathrm{O}$ atoms) clearly showed that the $\mathrm{O}-\mathrm{O}$ separation was that of a dioxygen molecule and the separation was restrained to be within $0.02 \AA$ of the theoretical value. Solvent molecules were added in the final stages if they were clearly shown in $\left(F_{\mathrm{o}}-F_{\mathrm{c}}\right)$ syntheses at a level $\geq 3.5$ r.m.s., were located in chemically sensible positions and refined to give thermal isotropic coefficients $<70 \AA^{2}$. The final model refined to an $R$ and $R_{\text {free }}$ of 0.178 and 0.228 , respectively, in the resolution range $68.5-2.8 \AA$ and was validated using the program PROCHECK (Laskowski et al., 1993) as indicated in Table 2.

\section{Results and discussion}

\subsection{Overall organization of the molecule}

Fig. 1 shows the overall organization of the human ceruloplasmin (hCP) molecule and the locations of the various metal cations: $\mathrm{Cu}^{2+}, \mathrm{Ca}^{2+}$ and $\mathrm{Na}^{+}$. The molecule is comprised of six cupredoxin-type domains (Zaitseva et al., 1996) coloured red through to purple. Domains 1, 3 and 5 comprise some 190 amino-acid residues, whereas the even domains are smaller, with around 150 residues. Domains 2, 4 and 6 possess a binding site for a mononuclear type 1 copper centre; a trinuclear copper centre comprising a type 2 copper ion and two type 3 copper ions is located between domains 1 and 6 . Domain 1 has a $\mathrm{Ca}^{2+}$-binding site towards its outer surface. The lower surface of the molecule is relatively planar, with the exception of the loops connecting the domains, but the top surface has three large protuberances formed by loops from pairs of odd and even domains. Thus, the loop between $\beta$-strands 1 and 2 of an odd domain packs against the loop between $\beta$-strands 1 and 2 of an even domain and this association is assisted by a $\mathrm{Na}^{+}$ cation (see below).

The quality of the model is high for a $2.8 \AA$ resolution structure (Table 2), but some loops and inter-domain connecting strands are still either poorly defined or completely

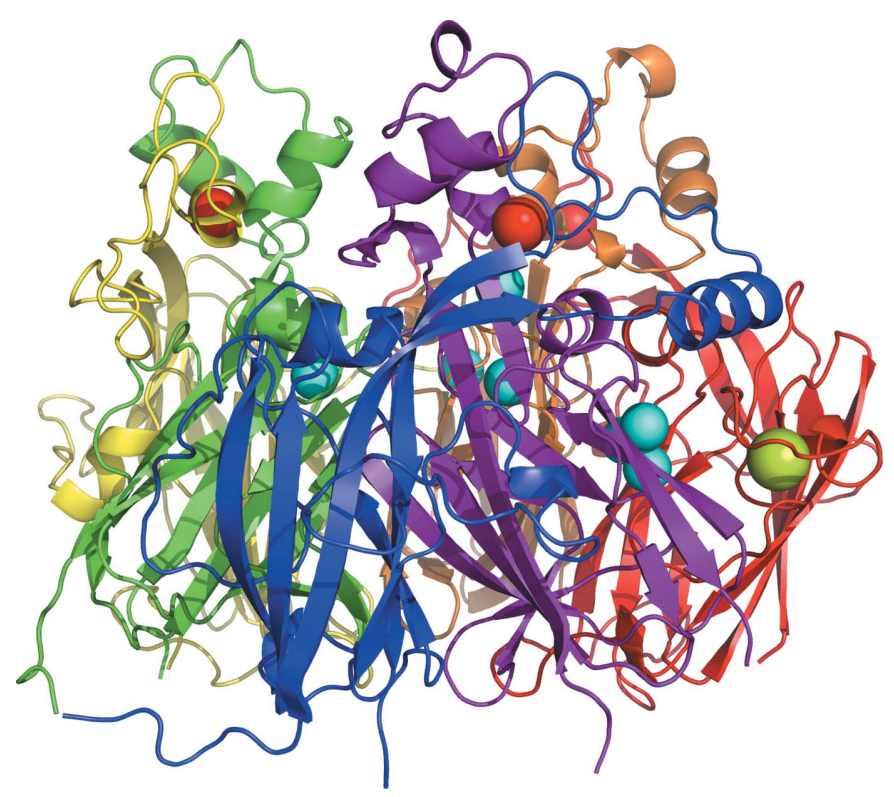

Figure 1

The overall organization of the ceruloplasmin molecule, showing the six cupredoxin domains (domains 1, 2, 3, 4, 5 and 6 in red, orange, yellow, green, blue and purple, respectively) and the locations of the metalbinding sites: $\mathrm{Cu}^{2+}$ as blue spheres, $\mathrm{Ca}^{2+}$ as an olive-green sphere and $\mathrm{Na}^{+}$ as red spheres. The relatively planar bottom surface and the protuberances at the top surface are clearly visible. The figures were prepared with the PyMOL program (DeLano, 2002). 
Table 3

Molecular geometry at the metal-binding sites: the mononuclear binding sites (T1 type copper and the second metal centre in domain 6) and ligands.

\begin{tabular}{|c|c|c|c|c|}
\hline Atom $A$ & Atom $B$ & $\begin{array}{l}A-B \text { distance } \\
(\AA)\end{array}$ & Atom $C$ & $\begin{array}{l}B-C \text { distance } \\
(\AA)\end{array}$ \\
\hline \multicolumn{5}{|l|}{ Domain 2} \\
\hline \multirow[t]{4}{*}{$\mathrm{Cu} 21$} & His $276 \mathrm{~N}^{\delta 1}$ & 2.02 & & \\
\hline & His $324 \mathrm{~N}^{\delta 1}$ & 1.88 & & \\
\hline & Cys319 $\mathrm{S}^{\gamma}$ & 1.94 & & \\
\hline & Leu329 $C^{\delta 1}$ & 3.52 & & \\
\hline \multicolumn{5}{|l|}{ Domain 4} \\
\hline \multirow[t]{4}{*}{$\mathrm{Cu} 41$} & His637 $\mathrm{N}^{\delta 1}$ & 2.08 & & \\
\hline & His $685 \mathrm{~N}^{\delta 1}$ & 2.01 & & \\
\hline & Cys680 S & 2.06 & & \\
\hline & Met690 S ${ }^{\delta}$ & 2.89 & & \\
\hline \multicolumn{5}{|l|}{ Domain 6} \\
\hline \multirow[t]{4}{*}{ Cu61 } & His975 $\mathrm{N}^{\delta 1}$ & 1.97 & & \\
\hline & His1026 $\mathrm{N}^{\delta 1}$ & 2.02 & & \\
\hline & Cys1021 S ${ }^{\gamma}$ & 2.14 & & \\
\hline & $1031 \mathrm{~S}^{\delta}$ & 3.32 & & \\
\hline \multirow[t]{5}{*}{$\mathrm{M} 62 \dagger$} & His $940 \mathrm{~N}^{\varepsilon 2}$ & 2.26 & & \\
\hline & Asp1025 $\mathrm{O}^{\delta 1} \dagger$ & 2.31 & & \\
\hline & W111 & 2.43 & Glu935 $\mathrm{O}^{\varepsilon 1}$ & 2.80 \\
\hline & W200 & 2.41 & Asp1025 $\mathrm{O}^{\varepsilon 2} \dagger$ & 2.96 \\
\hline & W292 & 2.24 & Glu272 $\mathrm{O}^{\varepsilon 1}$ & 3.18 \\
\hline
\end{tabular}

$\dagger$ M62 and Asp1025 were assigned an occupancy of 0.5.

lacking electron density. Thus, there is a break in the main chain between residues 476 and 482 in domain 3 corresponding to the formation of the 67 and $50 \mathrm{kDa}$ fragments (Takahashi et al., 1983) and the interconnecting region between domains 5 and 6 , residues 885-888, and the last few residues at the C-terminus, 1041-1046, are not defined. Three further regions, 18-32 in domain 1 and the regions connecting domains 2 and 3 (residues 340-350) and 4 and 5 (residues 703709), are poorly defined, but are included in the model on a 'best efforts' basis. For the remainder of the structure, $86.7 \%$ of the residues (749) lie in the most favoured regions of a Ramachandran plot (Ramakrishnan \& Ramachandran, 1965), $12.6 \%$ (109) lie in additionally allowed regions, $0.6 \%$ (5) lie in generously allowed regions and $0.1 \%$ (1) lie in disallowed regions. The last category involves residue $\operatorname{Arg} 883$, which is close to the missing region between domains 5 and 6 .

$\mathrm{hCP}$ is known to show heterogeneity with respect to its glycan moieties, but usually possesses at least three glycan chains terminating in sialic acid residues (Yamashita et al., 1981; Endo et al., 1982). In the predominant heteromer, two biantennary and one triantennary chain are $\mathrm{N}$-linked to asparagine residues located within the $67 \mathrm{kDa}$ fragment of the molecule at Asn119, Asn339 and Asn378. A fourth chain that is also triantennary can be attached to Asn743. In the current X-ray model, only the first units of the glycan chains at Asn119 and Asn378 are clearly visible.

\subsection{The mononuclear $\mathrm{T} 1$ copper-binding sites}

hCP contains three mononuclear T1-type (Malmström, 1982) copper-binding sites located in the even domains 2,4 and 6 . The T1 sites in domains 4 and 6 are typical blue-copper sites with two histidines and a cysteine ligand at around $2.0 \AA$ and a fourth weaker ligand, a methionine, at distance of around $3.0 \AA$ (Table 3 ). The mononuclear site in domain 2 is similar to that found in several fungal laccases (Ducros et al., 2001; Piontek et al., 2002; Garavaglia et al., 2004) in that it lacks the weak methionine ligand, which is replaced by an aliphatic leucine residue at a closest distance of around $3.5 \AA$. These three sites are not significantly different from those described in detail for the room-temperature structure.

Ceruloplasmin is unique among the other multi-copper oxidases in that it contains three type 1 'integral' copper ions in the even-numbered domains, whereas only the T1 copper in domain 6 at a distance of some $12-13 \AA$ from the trinuclear cluster is absolutely necessary for ceruloplasmin to perform catalytic action according to the schemes suggested for ascorbate oxidase and laccase (Messerschmidt et al., 1992; Bento et al., 2005). The distances between the copper ions in the even domains of around $18 \AA$ is well within the range of effective electron transfer (Lindley et al., 1997; Machonkin \& Solomon, 2000) and it has been suggested that the copper ions in domains 2 and 4 could provide a slower route of electron transfer, thus permitting the oxidation of more than one substrate molecule at a time. This could in turn accomplish a more effective transfer of four electrons to the dioxygen molecule from the reducing substrate. The role of the copper ion in domain 2, however, remains intriguing. Firstly, none of the metal-soaking or organic substrate-soaking experiments gave any indication that reducing substrates bind in domain 2 . Secondly, the replacement of the axial ligand of the T1 copper in domain 2, Leu329, with Met by site-directed mutagenesis resulted in a silent mutation in that it failed to modify either the spectroscopic or catalytic properties of the mutated protein (Bielli et al., 2001). Whether the T1 site in domain 2 is an 'evolutionary relic' and therefore functionally silent or

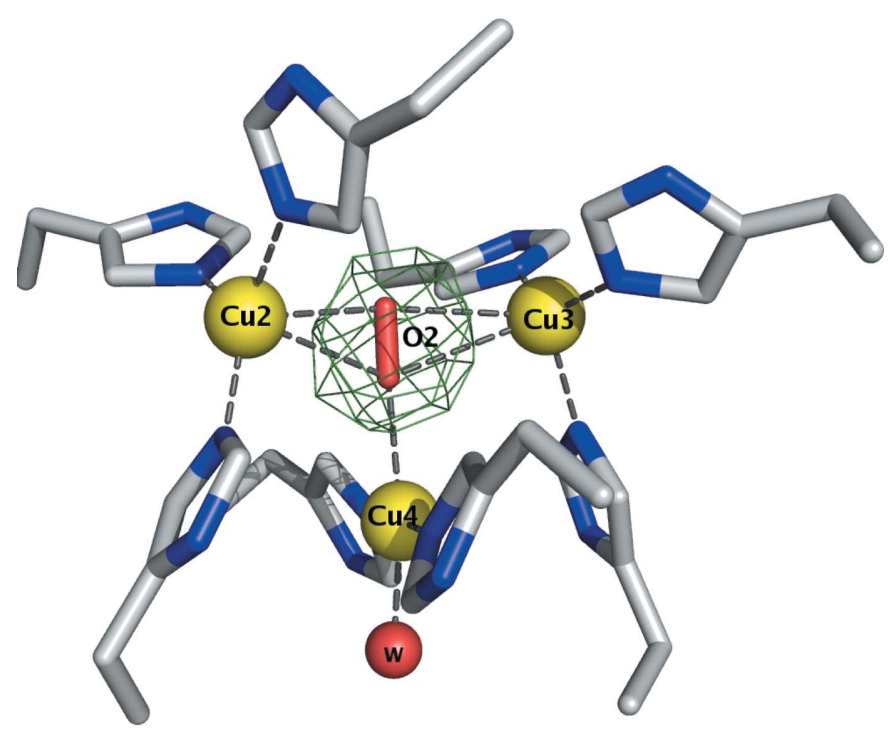

Figure 2

The trinuclear cluster between domains 1 and 6 , showing the water molecule attached to the type 2 copper $\mathrm{Cu} 34$. An OMIT electron-density map, $F_{\mathrm{o}}-F_{\mathrm{c}}$, contoured at 7 r.m.s. clearly confirms the presence of a diatomic species, assumed to be dioxygen, within the type 3 cluster. 
whether this copper ion is destined for other functions still remains unclear.

\subsection{The trinuclear copper centre}

The trinuclear copper centre comprising two type 3 copper cations and a type 2 cation may well contain a mixture of states, but the dominant species is that with a diatomic species bound to the trinuclear centre as shown in Fig. 2. The separation of the two type 3 copper cations, $\mathrm{Cu} 2-\mathrm{Cu} 3=$ $4.97 \AA$, readily accommodates this species and it has been modelled as a dioxygen molecule in a similar manner to that found in the Bacillus subtiltis endospore coat protein (Bento et al., 2005). Details of the molecular geometry are given in Table 4. A network of solvent molecules is found in the entrance channel to the trinuclear site, but some of these are weakly defined and may not have full occupancies. Thus, W199 is 2.59 and $2.43 \AA$ from $\mathrm{Cu} 2$ and $\mathrm{O} 2$ of the dioxygen moiety, respectively, and also is hydrogen bonded to $\mathrm{W} 1$ and $\mathrm{W} 2$ at 3.12 and $2.19 \AA$, respectively. W1 is $3.07 \AA$ from W195, which in turn is hydrogen bonded to the $\mathrm{O}^{\varepsilon 1}$ atom of Glu1032 $\left(\mathrm{W} 195-\mathrm{O}^{\varepsilon 1}=2.74 \AA\right.$ ) and $\mathrm{W} 123$ at $3.03 \AA$; W123 is $3.10 \AA$ from the $\mathrm{O}^{\varepsilon 1}$ atom of Gln740. Glu1032 may be the acidic residue necessary for the initial protonation of the dioxygen molecule via the water network. In CotA the equivalent of Glu1032 is Glu498, although the latter residue is on the opposite side of the entrance channel (equivalent to Ile1027 in hCP) and closer to the trinuclear centre.

The type 2 copper ion, $\mathrm{Cu} 4$, appears to have two configurations at around the $50 \%$ level, the first with an oxygen moiety O35, presumably hydroxide, at a distance of around $2.05 \AA$ and the second with a water molecule, W148, at a distance of $2.98 \AA$. In either case, a hydrogen bond is formed with the hydroxyl group of Tyr107 (W148-OH $=2.33 \AA$ ). W148 is also hydrogen-bonded to the main-chain carboxyl group of Ser102 (W148-O = 3.23 $\AA$ ) and W106 (W148$\mathrm{W} 106=2.62 \AA)$, which in turn forms hydrogen bonds to the $\mathrm{O}^{\delta 2}$ atom of Asp995 (Wat106- $\mathrm{O}^{\delta 2}=2.57 \AA$ ) and the mainchain carboxyl moiety of Phe979 (Wat106-O = $3.19 \AA$ ). According to the reaction scheme proposed by Bento et al. (Bento et al., 2005), both the hydroxyl group and the water molecule probably arise from reduction of a dioxygen at the trinuclear cluster.

\subsection{The additional metal-binding sites for $\mathrm{Cu}, \mathrm{Fe}$ and $\mathrm{Co}$}

In previous studies (Lindley et al., 1997), additional metal sites were identified in domains 4 and 6, roughly 9.0-10.0 distant from the respective mononuclear copper centres and therefore well within electron-transfer range. The sites involve a histidine residue and negatively charged aspartate and glutamate residues and in the native crystal structure appear to be partially occupied by copper ions with occupancies of around $50 \%$. Interestingly, an additional site was not found in domain 2, where the mononuclear copper ion may be in the fully reduced state (Machonkin et al., 1998) and the histidine is replaced by a tyrosine residue. Soaking experiments with $\mathrm{CoCl}_{2}, \mathrm{FeSO}_{4}, \mathrm{FeCl}_{3}$ and $\mathrm{CuSO}_{4}$ gave credence to the idea that
Table 4

Molecular geometry at the metal-binding sites: the trinuclear copper cluster at the interface of domains 1 and 6 .

\begin{tabular}{|c|c|c|}
\hline Atom $A$ & Atom $B$ & Distance $(\AA)$ \\
\hline \multirow[t]{7}{*}{$\mathrm{Cu} 2$} & His $163 \mathrm{~N}^{\varepsilon 2}$ & 2.14 \\
\hline & His $980 \mathrm{~N}^{\varepsilon 2}$ & 1.97 \\
\hline & His1020 $\mathrm{N}^{\varepsilon 2}$ & 2.02 \\
\hline & Dioxygen $\mathrm{O} 2$ & 2.64 \\
\hline & Dioxygen $\mathrm{O} 1$ & 2.77 \\
\hline & $\mathrm{Cu} 3$ & 4.97 \\
\hline & $\mathrm{Cu} 4$ & 4.02 \\
\hline \multirow[t]{6}{*}{$\mathrm{Cu} 3$} & His103 $\mathrm{N}^{\varepsilon 2}$ & 1.90 \\
\hline & His161 $\mathrm{N}^{\varepsilon 2}$ & 2.09 \\
\hline & His1022 $\mathrm{N}^{\varepsilon 2}$ & 2.20 \\
\hline & Dioxygen $\mathrm{O} 2$ & 2.36 \\
\hline & Dioxygen $\mathrm{O} 1$ & 2.58 \\
\hline & $\mathrm{Cu} 4$ & 4.09 \\
\hline \multirow[t]{5}{*}{$\mathrm{Cu} 4$} & His101 $\mathrm{N}^{\varepsilon 2}$ & 1.87 \\
\hline & His $978 \mathrm{~N}^{\varepsilon 2}$ & 2.00 \\
\hline & Dioxygen $\mathrm{O} 1$ & 2.25 \\
\hline & $\mathrm{OH} \dagger$ & 2.05 \\
\hline & W148† & 2.98 \\
\hline
\end{tabular}

$\dagger$ Assigned occupancies of 0.5 .

these additional sites were sites of metal oxidation. The existence of the iron-binding sites in domains 4 and 6 of the hCP structure appears to be consistent with the earlier biochemical data (Frieden \& Hsieh, 1976) suggesting two iron-binding sites in the enzyme. The access to these sites is limited to relatively small substrates by three large protuberances at the top of the hCP molecule (see §3.6). In the case of soaking with $\mathrm{CoCl}_{2}$, the metal appeared to replace any copper that was bound and to fill the additional sites in domains 4 and 6 . The sites are rich in oxygen donors and therefore amenable to the presence of $\mathrm{Co}^{2+}$, but the metal could also become oxidized to the kinetically inert $\mathrm{Co}^{3+}$ through the transfer of an electron to the respective mononuclear copper centres. For both $\mathrm{Fe}^{2+}$ and $\mathrm{Fe}^{3+}$, the metals appeared to bind to 'holding' sites near the outside of the protein and in the case of the $\mathrm{Fe}^{2+}$ this could be explained by the metal occupying the additional site, releasing an electron to the nearest mononuclear copper and then translocating to the holding site with Glu597 and Glu935 in domains 4 and 6 , respectively, playing key roles in the translocation process. At the holding site the ferric iron would be available for collection, for example, by serum transferrin.

In the present study, there is no clear indication of an additional copper site in domain 4, but rather a solvent molecule, W201, that forms hydrogen bonds with His602 $\left(\mathrm{W} 201-\mathrm{N}^{\varepsilon 2}=2.28 \AA\right)$, Glu971 (W201- $\mathrm{O}^{\varepsilon 2}=2.35 \AA$ ) and a second solvent molecule W321 at $2.44 \AA$. In turn, W321 forms a hydrogen bond with Glu597 (W321- $\mathrm{O}^{\varepsilon 2}=2.37 \AA$ ). In domain 6 , the additional copper site does appear to contain a metal cation but at a low occupancy of only around $50 \%$ and its nature is difficult to define precisely. As indicated in Table 3, there could be up to five ligands, three of which are water molecules, arranged in a distorted trigonal bipyramid. Asp1025 clearly shows two alternative configurations, one oriented towards the metal site and the second pointing away so that the $\mathrm{O}^{\delta 1}$ atom forms a $1-6$ inter-domain hydrogen bond with Asn $271 \mathrm{~N}^{\delta 2}$ at a distance of $2.90 \AA$. Although the addi- 
tional site in domain 6 is not fully defined, the observations appear to be consistent with it acting as a site of metal-cation oxidation and with Glu935 playing a role in translocating the oxidized metal ion to a holding site closer to the molecular surface (Lindley et al., 1997).

\subsection{The calcium-binding site}

The room-temperature structure determination of human ceruloplasmin at $3.1 \AA$ resolution indicated a binding site unique to domain 1 (residues 1-192 inclusive), although it was not possible at this resolution to determine the precise nature of the moiety that was bound. The cryo-data at $2.8 \AA$ resolution show refinement parameters consistent with a $\mathrm{Ca}^{2+}$ cation in an environment comprising two bidentate aspartate ligands, Asp127 and Asp128, the carboxyl main-chain oxygen moieties of Lys109 and Gln124 and two water molecules in an octahedral coordination as shown in Fig. 3; details of the molecular geometry are given in Table 5. The discovery of the $\mathrm{Ca}^{2+}$ binding site is consistent with binding studies of divalent cations to human and sheep ceruloplasmin, in which an effective one-step purification protocol based on the affinity of the protein for $\mathrm{Ca}^{2+}$ ions has been developed (Musci et al., 1996). This $\mathrm{Ca}^{2+}$-binding site is almost identical to that found in the structure of C-inactivated bovine factor Va (Adams $e t$ al., 2004); the respective sequences in this region are as follows, with the ligands to the $\mathrm{Ca}^{2+}$ ion shown in bold.

hCP 105 ITYYKEHEGA I YPDNTTDFQRA DDKVYPGE 135 Va 89 IKYSKFSEGASYSDHTLPMEKMDDAVAPGQ 118

Indeed, the A1 domain of factor Va can be superimposed on the motif comprising domains 1 and 2 of hCP with an r.m.s. fit of $1.36 \AA$ for 261 (of 340) $C^{\alpha}$ atoms.

In the case of the factor Va chain, association between the light and heavy chains is required for the protein to function and this has been shown to be dependent on a $\mathrm{Ca}^{2+}$ cation. However, the $\mathrm{Ca}^{2+}$ cation does not bridge the light and heavy chains; it is entirely coordinated in the A1 domain. It has been proposed that the loop comprising Lys93-Asp112 adopts a conformation that results in several essential interactions between the $\mathrm{A} 1$ and $\mathrm{A} 3$ domains, thereby mediating chain association. Lys93, Asp111 and Asp112 in factor Va are equivalent to Lys109, Asp127 and Asp128 in hCP, whereas the A3 domain in factor $\mathrm{Va}$ is equivalent to domains 5 and 6 of hCP. In hCP, Lys109 forms a hydrogen-bonding interaction with the carbonyl moiety of Ile1016 in domain 6 and the $\mathrm{Ca}^{2+}$ ion is $11.9 \AA$ from the type 2 copper of the trinuclear centre which lies between domains 1 and 6 . Indeed, the $\mathrm{Ca}^{2+}$ ion and type 2 copper can be linked through a pathway starting with Asp127 OD2, which is $2.93 \AA$ from the main-chain $\mathrm{N}$ of Glu112. The carbonyl $\mathrm{O}$ of Glu112 is then $2.79 \AA$ from His101 ND1, which in turn is bonded to $\mathrm{Cu} 4$ through its NE2 atom.

In the case of hCP, its interaction with red blood cells has been shown to be a $\mathrm{Ca}^{2+}$-dependent process and is limited by hCP binding to the cell membrane (Saenko \& Yaropolov, 1990), but not being transported through it. Further studies on
Table 5

Molecular geometry at the metal-binding sites: the $\mathrm{Ca}^{2+}$-binding site in domain 1.

\begin{tabular}{lll}
\hline Atom $A$ & Atom $B$ & Distance $(\AA)$ \\
\hline Ca & Lys109 O & 2.36 \\
& Gln124 O & 2.44 \\
& Asp127 OD1 & 2.48 \\
& Asp127 OD2 & 2.47 \\
& Asp128 OD1 & 2.52 \\
& Asp128 OD2 & 2.25 \\
& W17 & 2.33 \\
W17 & W69 & 2.53 \\
& Ala114 O & 2.70 \\
W69 & Glu112 OE1 & 3.03 \\
& Asp118 OD2 & 2.77 \\
& Thr120 OG1 & 2.85 \\
& Gln124 O & 2.76 \\
\hline
\end{tabular}

the nature of the interactions have shown that terminal sialic residues both on the red blood-cell receptors and the hCP glycan moieties play determining roles. Although the $\mathrm{Ca}^{2+}$ cation is entirely coordinated by residues in domain 1 , it is only some 7.0-8.0 $\AA$ from the protein surface and $10.0 \AA$ from the $\mathrm{C}^{\alpha}$ atom of Asn119, a residue to which a glycan moiety is $\mathrm{N}$-linked. It is therefore possible that the binding of a $\mathrm{Ca}^{2+}$ ion can mediate the conformation of at least one of the glycan moieties and that this is turn can play a role in the binding of hCP to red blood cells.

\subsection{The sodium-binding sites}

The molecule of ceruloplasmin possesses an upper surface with three large protuberances. These features are formed from the loops between $\beta$-strands 1 and 2 of one odd and one even domain as shown in Fig. 4(a) for the protuberance formed by domains 5 and 6 . It has been proposed that they help to limit access to the putative oxidation sites close to the T1 copper-binding sites in domains 4 and 6 (Lindley et al., 1997; Zaitsev et al., 1999). Thus, metal cations and species such as the biogenic amines norepinephrine, epinethrine, dopamine and serotonin will have access, whereas larger molecules will not. This could rationalize the function of $\mathrm{hCP}$ as a ferroxidase and/or its control over the concentration of biogenic amines reaching the blood-brain barrier. The locations of the T1 copper sites in the even domains that are oriented inside the protein as opposed to the odd domains that point towards the outside must also play an important role, but the rigidity of these structures will also be of importance. The hCP structure determined at cryotemperature identifies a metal-binding site near the base of each of the three protuberances that will greatly assist their rigidity. The behaviour of the isotropic thermal parameters of the metals during refinement and their chemical environment strongly suggests they are $\mathrm{Na}^{+}$cations. Indeed, the crystallization of the trigonal form of $\mathrm{hCP}$ has not been found to be possible in the absence of sodium chloride. The roles of $\mathrm{Na}^{+}$and $\mathrm{K}^{+}$ions in enzyme function have been comprehensively described (Page \& Di Cera, 2006), but in hCP the $\mathrm{Na}^{+}$ions appear to have a predominantly structural function. Thus, as shown in Fig. 4(b), the metal at the base of 
the protuberance formed by domains 5 and 6 has a squarepyramidal environment with a tyrosine ligand (Tyr760) and three carboxyl O atoms (residues Phe748, Gly757 and Ser936) at its base and a water molecule (W64) in the apical position. Met939 $\mathrm{S}^{\delta}$ is some $3.47 \AA$ distant on the other side of the base to the water molecule. The water molecule is in turn tetrahedrally coordinated to two further waters W115 and W129 and the peptide $\mathrm{N}$ atom of Gly757. W115 forms hydrogen bonds with Ser936 $\mathrm{O}^{\gamma}$ and Asn93 $\mathrm{O}^{\delta 1}$, whereas W129 hydrogen bonds to the carboxyl oxygen moieties of Ala747 and Asp750. This system of covalent and hydrogen bonding ensures that the large loops between the first two $\beta$-strands of both domains 5 and 6 form a compact and stable arrangement (Fig. 4a). As indicated in Table 6, an almost identical situation

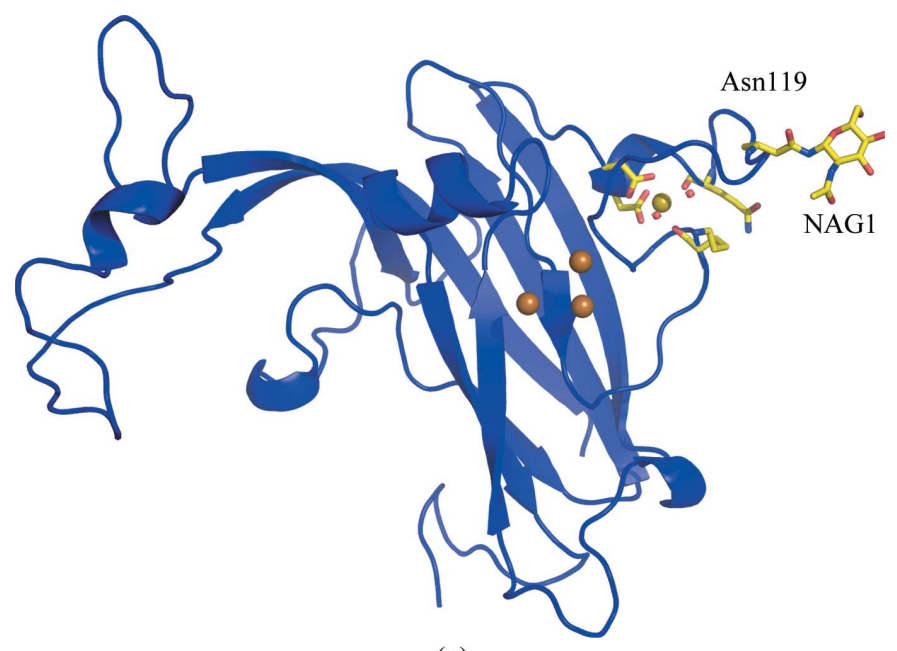

(a)

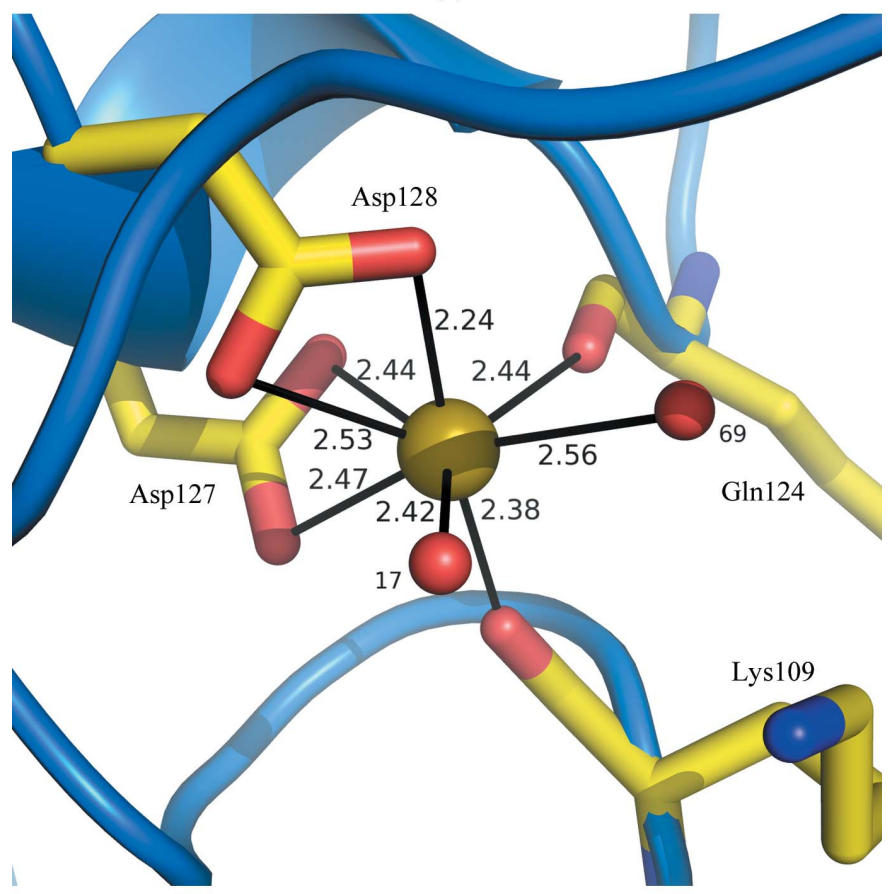

(b)

Figure 3

The $\mathrm{Ca}^{2+}$-binding site in domain 1. (a) Overall view of domain 1, showing the locations of the trinuclear copper cluster and the glycan chain at Asn119. (b) Details of the molecular geometry at the $\mathrm{Ca}^{2+}$ centre. is found for domains 3 and 4, with the addition of a further hydrogen bond for W171 (equivalent to W115 in the domain $5 / 6$ interface), which forms an extra hydrogen bond to the peptide $\mathrm{N}$ atom of Ile397. However, the arrangement at the base of the protuberance formed by domains 1 and 2 is less well defined, mirroring the overall poorer definition of domain 1 with respect to the other five domains. Not only is Met240 S ${ }^{\delta}$ appreciably further away from the $\mathrm{Na}^{+}$cation $(3.85 \AA)$ relative to the other two $\mathrm{Na}^{+}$cations, but the water bound to it and a second water equivalent to W129 in Fig. 4(b) are also missing.

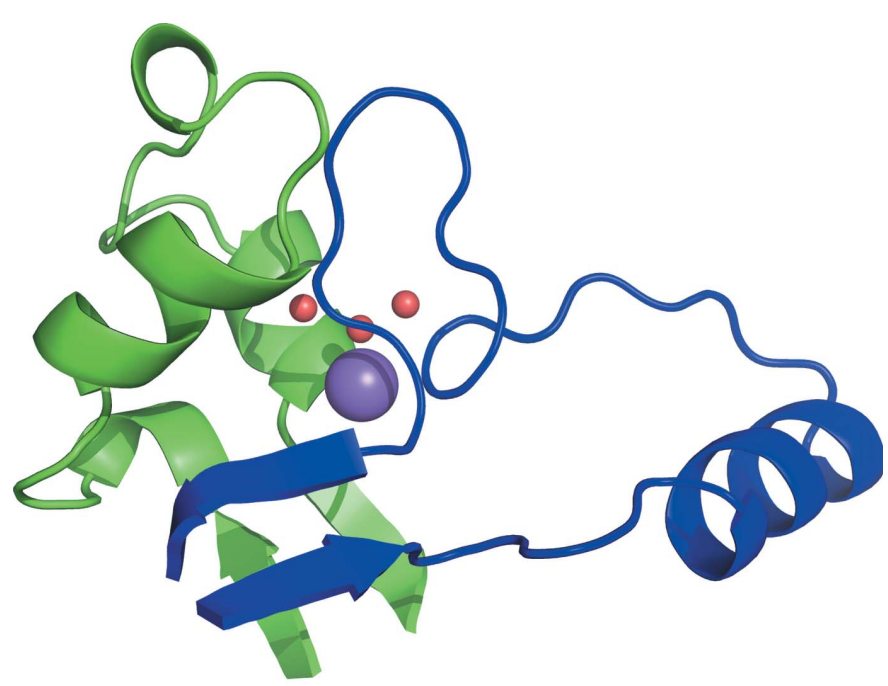

(a)

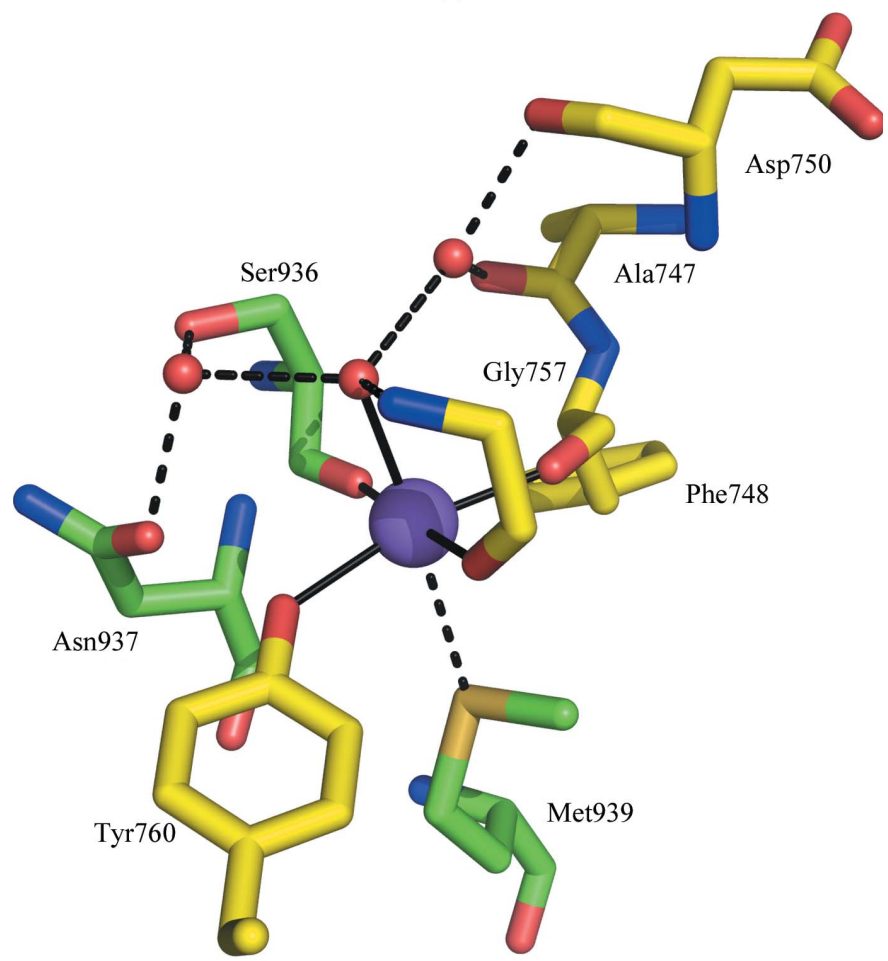

(b)

Figure 4

The $\mathrm{Na}^{+}$-binding site between domains 5 and 6. (a) An overall view with the main chains of domains 5 and 6 coloured blue and green, respectively. (b) A detailed view of the bonding in the vicinity of the Na4 cation site. In (b) Ile756 is not shown for clarity, although its main-chain $\mathrm{N}$ atom forms a hydrogen bond with W115 on the left-hand side of the figure (see Table 6). 
Table 6

Molecular geometry at the metal-binding sites: the $\mathrm{Na}^{+}$-binding sites between the odd and even domains.

Even-domain residues are in bold.

\begin{tabular}{|c|c|c|c|c|c|c|c|c|}
\hline \multicolumn{3}{|c|}{ Domains 1 and 2} & \multicolumn{3}{|c|}{ Domains 3 and 4} & \multicolumn{3}{|c|}{ Domains 5 and 6} \\
\hline$A$ & $B$ & $A-B(\AA)$ & $A$ & $B$ & $A-B(\AA)$ & $A$ & $B$ & $A-B(\AA)$ \\
\hline \multirow{8}{*}{$\mathrm{Na} 2$} & Tyr48 OH & 2.44 & $\mathrm{Na} 3$ & Tyr401 OH & 2.30 & $\mathrm{Na} 4$ & Tyr760 OH & 2.46 \\
\hline & Ser237 O & 2.43 & & Ser598 o & 2.35 & & Ser936 O & 2.32 \\
\hline & Gly45 O & 2.48 & & Gly398 O & 2.41 & & Gly757 O & 2.52 \\
\hline & & & & W159 & 2.43 & & W64 & 2.44 \\
\hline & Met240 S $\delta$ & 3.85 & & Met601 S $\mathbf{S}^{\delta}$ & 3.36 & & Met939 $\mathrm{S}^{\delta}$ & 3.40 \\
\hline & & & & Gly398 N & 2.98 & & Gly757 N & 2.84 \\
\hline & & & W45 & Val388 O & 2.96 & W129 & Ala747 O & 2.93 \\
\hline & & & & Glu391 O & 2.58 & & Asp750 O & 2.77 \\
\hline \multirow[t]{3}{*}{ W40 } & Ile44 N & 3.01 & W171 & Ile397 N & 2.95 & W115 & Ile756 N & 3.24 \\
\hline & Ser237 $\mathbf{O}^{\gamma}$ & 2.70 & & Ser598 O ${ }^{\gamma}$ & 2.83 & & Ser396 $0^{\gamma}$ & 2.63 \\
\hline & Asn238 $0^{\delta 1}$ & 2.81 & & $\operatorname{Asn599} 0^{\delta 1}$ & 2.64 & & $\operatorname{Asn937} 0^{\delta 1}$ & 2.62 \\
\hline
\end{tabular}

This could be taken as an indication of lower rigidity for this structure and could correlate with the copper ion in domain 2 being in the fully reduced state (Machonkin et al., 1998) and the absence of any additional metal-binding site in this domain. In this context, it should also be noted that the $\mathrm{Na}^{+}$ ion between domains 5 and 6 is relatively close to the additional binding site in domain 6 . In this cryo-structure, the cation is liganded to the main chain of Ser936 which is adjacent to Glu935 and in turn is linked to the additional metal-binding site in domain 6 through W111. As indicated previously, Glu935 has been implicated in translocating metal ions to a holding site near the surface of the protein after oxidation. The $\mathrm{Na}^{+}$ion in domain 6 (and also domain 4) may therefore not only maintain the conformation of the upper surface protuberance, but may also be involved in the processes of metal oxidation and translocation.

\section{Summary}

The preparation of crystals of human ceruloplasmin that can be flash-frozen to liquid-nitrogen temperature has resulted in the collection of X-ray diffraction data to $2.8 \AA$ at a temperature of $100 \mathrm{~K}$, a marginally higher resolution, but certainly a significantly better quality data set than can be obtained at ambient temperatures. This has resulted in a structure with an overall better definition of the protein side chains, a re-examination of the trinuclear copper cluster with a bound dioxygen moiety and the identification of $\mathrm{Ca}^{2+}$ and $\mathrm{Na}^{+}$ centres. The $\mathrm{Ca}^{2+}$ centre appears to be an integral component of the structure and may play a role in the interactions of the protein with red blood cells. The presence of sodium chloride in the crystallization medium is essential to obtain the trigonal crystals of hCP used in this analysis. The $\mathrm{Na}^{+}$ions appear to play a crucial role not only in the overall fold of the protein, but also in the formation of structural elements that restrict access to the type 1 copper sites.

The presence of these centres, together with previous studies on the binding of various transition metals, biogenic amines and aromatic diamines, further emphasizes the prob- ability that ceruloplasmin is multi-functional. The multifunctional nature of oxidase activity of ceruloplasmin has also been revealed in anion-binding studies (Musci et al., 1999). In contrast to cations, the anions (azide and $\mathrm{Cl}^{-}$) appear to act either as inhibitors or activators, thus modulating the oxidase activity of the enzyme, the effect of the anions being concentration-dependent and $\mathrm{pH}$-dependent.

It should be remembered that the protein is an acute phase entity so that its concentration in the plasma increases twofold to threefold at the onset of infection or wounding. Under these circumstances, the multi-functionality may be highly important. However, there is no direct evidence from the structural viewpoint that the protein plays a dominant role in copper transport. Whilst copper can bind to the additional binding sites in domains 4 and 6 and presumably to other sites on the complex surface of this large protein and whilst catabolism of the protein will result in the integral mononuclear and trinuclear copper loaded in the hepatic liver cells being released into the general environment, the protein does not have a structure that is typical of other molecules involved in copper transport (Banci et al., 2006). The case for ceruloplasmin as a copper transporter appears to be, as yet, unresolved.

Future work will focus on obtaining larger crystals with the hope that this will lead to an even higher resolution definition of the ceruloplasmin structure.

We wish to acknowledge the European Synchrotron Radiation Facility in Grenoble, France for the provision of X-ray data-collection facilities and the assistance of the Macromolecular Crystallography project team in helping to optimize the data quality on beamline ID14-4. We would also like to thank the Laboratory of Animal Cell Technology (Professor Manuel Carrondo and Dr Paula Alves) at the Instituto de Biologia Experimental e Tecnológica for help with the purification of the hCP. The Fundação para a Ciência e a Tecnologia, Portugal is also thanked for providing support to IB. 


\section{References}

Adams, T. E., Hockin, M. F., Mann, K. G. \& Everse, S. J. (2004). Proc. Natl Acad. Sci. USA, 101, 8918-8923.

Banci, L., Bertini, I., Cantina, F., DellaMalva, N., Herrmann, T., Rosato, A. \& Wuthrich, K. (2006). J. Biol. Chem. 281, 29141-29147.

Bento, I., Martins, L. O., Lopes, G. G., Carrondo, M. A. \& Lindley, P. F. (2005). Dalton Trans. 21, 3507-3513.

Bielli, P., Bellenchini, G. C. \& Calabrese, L. (2001). J. Biol. Chem. 276, 2678-2685.

Bielli, P. \& Calabrese, L. (2002). Cell. Mol. Life Sci. 59, 1413-1427.

Collaborative Computational Project, Number 4 (1994). Acta Cryst. D50, 760-763.

DeLano, W. L. (2002). The PyMOL Molecular Visualization System. DeLano Scientific, San Carlos, CA, USA. http://www.pymol.org.

Ducros, V., Brzozowski, A. M., Wilson, K. S, Ostergaard, P., Schneider, P., Svendson, A. \& Davies, G. J. (2001). Acta Cryst. D57, 333-336.

Emsley, P. \& Cowtan, K. (2004). Acta Cryst. D60, 2126-2132.

Endo, M., Susuki, K., Schmidt, K., Fournet, B., Karamanos, Y., Montrueil, J., Dorland, L., Van Halbeek, H. \& Vliegenthart, J. F. G. (1982). J. Biol. Chem. 257, 8755-8760.

Frieden, E. \& Hsieh, H. S. (1976). Adv. Enzymol. Relat. Areas Mol. Biol. 44, 187-236.

Garavaglia, S., Cambria, M. T., Miglio, M., Ragusa, S., Iacobazzi, V., Palmieri, F., D'Ambrosio, C., Scaloni, A. \& Rizzi, M. (2004). J. Mol. Biol. 342, 1519-1531.

Holmberg, G. C. (1944). Acta Physiol. Scand. 8, 227-229.

Jones, T. A., Zou, J.-Y., Cowan, S. W. \& Kjeldgaard, M. (1991). Acta Cryst. A47, 110-119.

Laskowski, R. A., MacArthur, M. W., Moss, D. S. \& Thornton, J. M. (1993). J. Appl. Cryst. 26, 283-291.

Laurie, S. N. \& Mohammed, E. H. (1980). Coord. Chem. Rev. 33, 279-312.

Leslie, A. G. W. (1992). Joint CCP4/ESF-EACBM Newsl. Protein Crystallogr. 26.

Lindley, P. F. (2001). Handbook on Metalloproteins, edited by I. Bertini, A. Sigel \& H. Sigel, pp. 763-811. New York: Marcel Dekker.

Lindley, P. F., Card, G., Zaitseva, I. \& Zaitsev, V. (1999). Perspectives on Bioinorganic Chemistry, Vol. 4, edited by R. W. Hay, J. R. Dilworth \& K. W. Nolan, pp. 51-89. Greenwich, CT, USA: JAI Press.
Lindley, P. F., Card, G., Zaitseva, I., Zaitsev, V., Reinhammer, B., Selin-Lindgren, E. \& Yoshida, K. (1997). J. Biol. Inorg. Chem. 2, 454-463.

Machonkin, T. E. \& Solomon, E. I. (2000). J. Am. Chem. Soc. 122, 12547-12560.

Machonkin, T. E., Zhang, H. H., Hedman, B., Hodgson, K. O. \& Solomon, E. I. (1998). Biochemistry, 37, 9570-9578.

Malmström, B. G. (1982). Ann. Rev. Biochem. 51, 21-59.

Messerschmidt, A., Ladenstein, R., Huber, R., Bolognesi, M., Avigliano, L., Petruzzelli, R., Rossi, A. \& Finazzi-Agro, A. (1992). J. Mol. Biol. 224, 179-205.

Moshkov, K. A., Lakatos, S., Hajdu, J., Zavodsky, P. \& Neifakh, S. A. (1979). Eur. J. Biochem. 94, 127-134.

Murshudov, G. N., Vagin, A. A., Lebedev, A., Wilson, K. S. \& Dodson, E. J. (1999). Acta Cryst. D55, 247-255.

Musci, G., Bellenchi, G. C. \& Calabrese, L. (1999). Eur. J. Biochem. 265, 589-597.

Musci, G., Bonaccorsi di Patti, M. C., Petruzzelli, R., Giartosio, A. \& Calabrese, L. (1996). Biometals, 9, 66-72.

Nakamura, K. \& Go, N. (2005). Cell. Mol. Life Sci. 62, 2050-2066.

Page, M. J. \& Di Cera, E. (2006). Physiol. Rev. 86, 1049-1092.

Piontek, K., Antorini, M. \& Choinowski, T. (2002). J. Biol. Chem. 277, 37663-37669.

Ramakrishnan, C. \& Ramachandran, G. N. (1965). Biophys. J. 5, 909-933.

Read, R. J. (1986). Acta Cryst. A42, 140-149.

Ryden, L. (1984). Copper Proteins and Copper Enzymes, Vol. 3, edited by R. Lontie, pp. 37-100. Boca Raton, FL, USA: CRC Press.

Saenko, E. L. \& Yaropolov, A. I. (1990). Biochem. Int. 20, 212-225.

Takahashi, N., Bauman, R. A., Ortel, T. L., Dwulet, F. E., Wang, C. C. \& Putnam, F. W. (1983). Proc. Natl Acad. Sci. USA, 80, 115-119.

Takahashi, N., Ortel, T. L. \& Putnam, F. W. (1984). Proc. Natl Acad. Sci. USA, 81, 390-394.

Vagin, A. \& Teplyakov, A. (1997). J. Appl. Cryst. 30, 1022-1025.

Yamashita, K., Liang, C. J., Funakoski, S. \& Kobata, A. (1981). J. Biol. Chem. 256, 1283-1289.

Yoshida, K,. Furihata, K., Takeda, A., Nakamura, A., Yamamoto, K., Morita, H., Hiyamuta, S., Ikeda, S., Shimizu, N. \& Yanagisawa, N. (1995). Nature Genet. 9, 267-272.

Zaitsev, V. N., Zaitseva, I., Papiz, M. \& Lindley, P. F. (1999). J. Biol. Inorg. Chem. 4, 579-587.

Zaitseva, I., Zaitsev, V., Card, G., Moshkov, K., Bax, B., Ralph, A. \& Lindley, P. (1996). J. Biol. Inorg. Chem. 1, 15-23. 